\title{
De la récurrence à la norme : interpréter les pratiques funéraires en préhistoire
}

\section{From repetition to norm: interpreting prehistoric funerary practices}

\author{
F. Bocquentin $\cdot$ P. Chambon $\cdot$ I. Le Goff $\cdot$ J. Leclerc $\cdot$ J.-G. Pariat $\cdot$ G. Pereira $\cdot$ C. Thevenet $\cdot$ F. Valentin
}

Reçu le 16 juillet 2009 ; accepté le 13 avril 2010

C Société d'anthropologie de Paris et Springer-Verlag France 2010

Résumé Pour aborder le discours des hommes sur la mort, le préhistorien est réduit à traquer des gestes réalisés autour des morts qu'il découvre : afin d'éviter toute surinterprétation, nous les appelons pratiques, les « pratiques funéraires ». Or, ce que nous souhaitons, c'est passer de la description à l'interprétation. Car ce qui a un sens, une logique, ce n'est pas un geste ou une collection de gestes, mais un système. Cependant, pour décoder un tel système, il faut d'abord être conscient que les gestes ne relèvent pas tous de l'obligation : à côté du « devoir-faire », la norme, il existe un «pouvoirfaire », ce qui est laissé à l'appréciation des exécutants. Sur le plan archéologique, il faut patiemment ordonner les pratiques : identifier leur récurrence, de jamais à toujours, ainsi que les corrélations entre les éléments constitutifs de la tombe. La difficulté consiste évidemment à passer de la dimension archéologique à la dimension funéraire initiale. À partir d'exemples tirés de nos recherches individuelles,

F. Bocquentin $\cdot$ P. Chambon $(\bowtie) \cdot$ F. Valentin

CNRS - UMR 7041, ethnologie préhistorique, MAE,

21, allée de 1'Université, F-92023 Nanterre cedex, France

e-mail : philippe.chambon@mae.u-paris10.fr

I. Le Goff

INRAP - UMR 7041, ethnologie préhistorique, MAE,

21, allée de 1'Université, F-92023 Nanterre cedex, France

J. Leclerc

UMR 7041, ethnologie préhistorique, MAE,

21, allée de l'Université, F-92023 Nanterre cedex, France

J.-G. Pariat

SDAVO - UMR 7041, ethnologie préhistorique, MAE,

21, allée de l’Université, F-92023 Nanterre cedex, France

G. Pereira

CNRS - UMR 8096, archéologie des Amériques, MAE,

21, allée de l'Université, F-92023 Nanterre cedex, France

C. Thevenet

UMR 7041, Protohistoire européenne, MAE,

21, allée de l'Université, F-92023 Nanterre cedex, France nous avons exploré trois directions. La définition de la norme dans un espace géographique nous a conduits à nous interroger sur les critères qui définissent nos ensembles. Au sein d'un ensemble défini, nous avons questionné la valeur que l'on doit accorder aux variations. Enfin, sur le plan diachronique, nous nous sommes penchés sur la permanence de la norme ou son changement, et donc sur les interactions entre la norme funéraire et les autres pans des activités sociales.

Mots clés Archéologie de la mort · Sépultures · Pratiques funéraires $\cdot$ Identités $\cdot$ Idéologie $\cdot$ Norme

Abstract Understanding societal conceptions of death presents a unique challenge to prehistorians because they cannot witness first-hand the funerary practices that interest them. Rather, they are limited to studying the physical traces left behind by such practices. However, we wish to move beyond description towards interpretation, because what interests us is not simply the individual practices (or even sets of practices), but the system that unifies them and imbues them with meaning. To unravel these systems, it is important to bear in mind that not all of the acts documented in the material record were obligatory. While there is the "devoir-faire" (what must be done), there also exists the "pouvoir-faire" (what can be done). The archaeologist must carefully categorize these practices: identify their frequency (from what is never done to what is always done), as well as make correlations among the essential elements of the burial. The obvious difficulty emerges when it is time to connect the archaeological interpretation to the reality of the original funerary practices. We explore three approaches that are drawn from our individual research. The definition of the norm within a given region leads us to question more closely the criteria by which groups are defined. Next, we explore the range of variability that can be reasonably expected within a single group. Finally, we examine the degree to which norms either remain stable or change through time, as 
well as the interactions between funerary practices and other aspects of the society.

Keywords Archaeology of death $\cdot$ Burials $\cdot$ Funerary practices · Identities · Ideology $\cdot$ Norm

\section{Abridged version}

Understanding societal conceptions of death presents a unique challenge to prehistorians because they cannot witness first-hand the funerary practices that interest them. Rather, they are limited to studying the physical traces left behind by such practices. However, we wish to move beyond description towards interpretation, because what interests us is not simply the individual practices (or even sets of practices), but the system that unifies them and imbues them with meaning.

To unravel these systems, it is important to bear in mind that not all of the acts documented in the material record were obligatory. While there is the "devoir-faire" (what must be done), there also exists the "pouvoir-faire" (what can be done). The archaeologist must carefully categorize these practices: identify their frequency (from what is never done to what is always done) as well as make correlations among the essential elements of the burial. The obvious difficulty emerges when it is time to connect the archaeological interpretation to the reality of the original funerary practices. If an act can be clearly identified as compulsory within a corpus being studied, an occurrence rate below $100 \%$ can be understood as a failure to respect an obligation, an indirect connection with an obligation, an unrecognised obligation for lack of correlation (for instance a change of scale), or simply because of fashion. The perception of funerary acts, in prehistoric archaeology, splits the data into two groups open to distinct interpretation: first, obligations which concern the entire sample or a clearly identified part of it; second, choices as well as obligations that go unrecognised because they concern a part of the sample which was not identified.

Three approaches are drawn from individual research, the examples being diverse both spatially and chronologically. The definition of the norm within a geographical area led to closer examination of the criteria used to define the groups. Defining the subject of research is a key difficulty given that prehistoric cultures have a vague relationship to the populations. The first solution is to proceed by successive approximations, confronting a posteriori the defined group with the cultural criteria. The second solution is to map out the spatial and chronological correspondence between the various indices. Sometimes the limits are clear and there is a veritable frontier between two norms because two conceptions conflict.
Within a well defined funerary area the value that should be accorded to variations is open to question. This research deals with finding what is the norm and what is not. A statistical approach to funerary practice has revealed a gradation in the representation. There are identities at different scales, from the individual to the group, from the regional to the global.

Finally, permanence or change in norms has been examined diachronically, as have the interactions between the funerary norm and other parts of society. The world of death was a place of broad social expression and, as one would expect, other norms coexisted with the funerary. These external stimuli, which did or did not cause changes or restructuration of funerary norms, are crucial when considering the articulation between the component parts. Such questions are at the heart of our research, as looking for what had to be done versus what could be done in prehistoric funerary practices, is only of interest when these differences can be interpreted, because what is being sought are funerary systems and the ideas which drove them.

\section{Introduction}

Cet article émane d'un travail collectif préliminaire mené par des chercheurs de la Maison de l'archéologie et de l'ethnologie de Nanterre autour de la notion de normes funéraires. À partir de nos terrains de recherche respectifs, éloignés tant du point de vue géographique que du point de vue chronologique, nous avons cherché les modalités de reconnaissance des obligations observées dans le traitement des défunts. Au-delà de l'identification des pratiques funéraires passées, la recherche des normes funéraires permet d'approcher l'idéologie des sociétés préhistoriques et, de ce fait, l'identité d'un ensemble culturel. Elle permet aussi d'aborder la nature et le rythme de son évolution. Il n'est ni possible ni légitime de s'engager dans cette voie à partir d'un site particulier : seule l'étude groupée et comparée d'un ensemble de sites permet d'écarter les comportements accidentels, facultatifs, ou anecdotiques, et de mettre en évidence constantes et régularités.

Le domaine funéraire se prête particulièrement bien à la recherche des normes, parce que celles-ci interviennent alors que le groupe traverse une crise qu'il tente d'endiguer par une procédure codifiée. Les pratiques funéraires lui permettent, d'une part d'organiser le devenir du mort, d'autre part de réaffirmer sa cohésion et sa pérennité ébranlées par cette disparition $[1,2]$.

Il faut reconnaitre d'emblée que les pratiques, dont nous pouvons observer archéologiquement la trace matérielle, ne concernent pas la totalité des défunts, ni même le plus souvent la majorité d'entre eux. Il reste qu'une pratique normée, même si elle est minoritaire, doit permettre de saisir quelque chose du système conceptuel sous-jacent. 


\section{De la norme funéraire à l'archéologie des normes funéraires}

\section{Définition de la norme : règle culturelle ou fréquence majoritaire ?}

Il est largement admis que dans une société donnée, il existe un ensemble de gestes funéraires que l'on peut qualifier de « normaux » et auxquels on oppose des pratiques qualifiées d'exceptionnelles, d'atypiques, singulières, voire de déviantes, ces dernières n'étant pas toujours liées à la sphère strictement funéraire ${ }^{1}$. Si ces traitements hors du commun ont récemment suscité un intérêt accru $[3,4]$, en revanche la " normalité » des pratiques funéraires est généralement admise comme une évidence.

L'interprétation des récurrences observées en archéologie en termes de norme ne va pourtant pas de soi. Elle se heurte à l'ambiguïté inhérente aux deux principales acceptions du mot «norme ». L'une fait de la norme un synonyme de la règle (Robert, TLF). L'autre a un sens statistique et définit la norme par rapport à la fréquence majoritaire (Littré, Robert, Dictionnaire de l'Académie, TLF). La première définition se fonde donc sur une perspective qualitative tandis que l'autre fait référence à un aspect quantitatif. Le choix de l'une ou de l'autre a des répercussions directes sur l'interprétation : si la première ne permet pas d'exclure qu'une pratique ne concernant que peu de sujets ne soit normée, la seconde conduit à considérer ces individus comme " hors norme » dans la mesure où leur traitement s'écarte de la majorité. Or, nombre de témoignages historiques ou ethnographiques le montrent : les pratiques funéraires, dont la structure s'apparente à un rite $[1,5]$, sont le résultat de comportements fortement codifiés, marqués d'obligations et frappés d'interdits, et relèvent ainsi de la première définition. Dans la mesure où notre objectif est de retrouver les règles qui régissent le traitement funéraire, c'est donc cette définition que nous retiendrons ici, la seconde ne correspondant qu'à une des expressions possibles (mais pas exclusives) des normes en vigueur. Dans le cas des sépultures archéologiques, faute d'avoir accès au système de valeur de la société que l'on étudie, l'assignation de tel ou tel geste funéraire à une norme relève donc d'une interprétation qui ne doit pas nous faire oublier la multiplicité des motivations qui déterminent ces gestes. Quelles sont les limites de cette interprétation ? Sous quelles conditions peut-on parler de normes? Dans quelle mesure certains gestes s'écartent-ils

\footnotetext{
${ }^{1}$ Elles peuvent certes s'inscrire dans un contexte funéraire comme c'est le cas des sépultures dites d'accompagnement, mais elles peuvent aussi relever d'autres formes de comportements mortuaires tels que le traitement pénal, sacrificiel, la privation de sépulture, culte des reliques, etc.
}

de ces normes ? Comment la norme varie-t-elle et se transforme-t-elle ? Autant d'interrogations que nous tentons d'illustrer dans la suite de cet article.

\section{Norme et écart à la norme : ce que l'on doit faire et ce que l'on peut faire}

Une fois exclues, les contraintes liées à l'environnement et au savoir-faire technique, on peut d'abord distinguer les gestes qui obéissent à une obligation de ceux qui relèvent d'un choix. Si l'on définit la norme funéraire comme un ensemble de conduites codifiées que les survivants sont tenus d'observer lors du traitement des défunts, elles appartiennent à la première de ces deux catégories. Qu'elles soient prescrites par une loi (écrite ou orale), induites indirectement par la pression sociale ou encore qu'elles aient été si bien intériorisées par l'habitude ou la tradition qu'elles semblent s'imposer à toutes comme " naturelles ", ces conduites font système. Elles peuvent être très contraignantes ou, au contraire, ne poser qu'un cadre assez lâche au sein duquel chaque individu ou groupe d'individus dispose d'une certaine liberté. Elles peuvent s'appliquer pareillement à tous les membres de la société (normes absolues) ou s'articuler en obligations distinctes (normes conditionnelles) pour certains segments de celleci (hommes/femmes ; nobles/roturiers ; bonne mort/mauvaise mort ; etc.). La norme peut ainsi se définir comme le « devoirfaire » qui regroupe l'ensemble des obligations et prohibitions constituant la structure élémentaire au sein de laquelle les pratiques funéraires doivent être effectuées (Fig. 1).

Cependant, tous les gestes funéraires ne sont pas conditionnés par la contrainte. Une part plus ou moins importante d'entre eux résulte d'un choix, qu'il soit choix individuel ou choix partagé par les membres d'un groupe social donné : c'est le « pouvoir-faire ».

Quelle qu'en soit la motivation, on observera une série de comportements qui peuvent rompre avec la norme en vigueur ou (le plus souvent) s'immiscer dans les espaces plus ou moins étendus qui échappent aux contraintes. Le choix peut être individuel et exprimer la volonté de personnaliser le traitement rendu au défunt ; il peut aussi exprimer l'adhésion à une mode ou tendance suivie par un groupe plus ou moins important de personnes [6]. Notons que cette tendance peut être largement répandue au sein d'une population quitte à s'ériger, en fin de compte, en une norme implicite si le même choix est opéré par tous.

\section{De la théorie à la pratique : problèmes de reconnaissance des règles et des choix en archéologie funéraire}

Percevoir la norme est donc rechercher des obligations : or celles-ci impliquent des gestes répétés. Ainsi, la norme absolue, c'est-à-dire qui ne dépend de rien et ne souffre d'aucune 


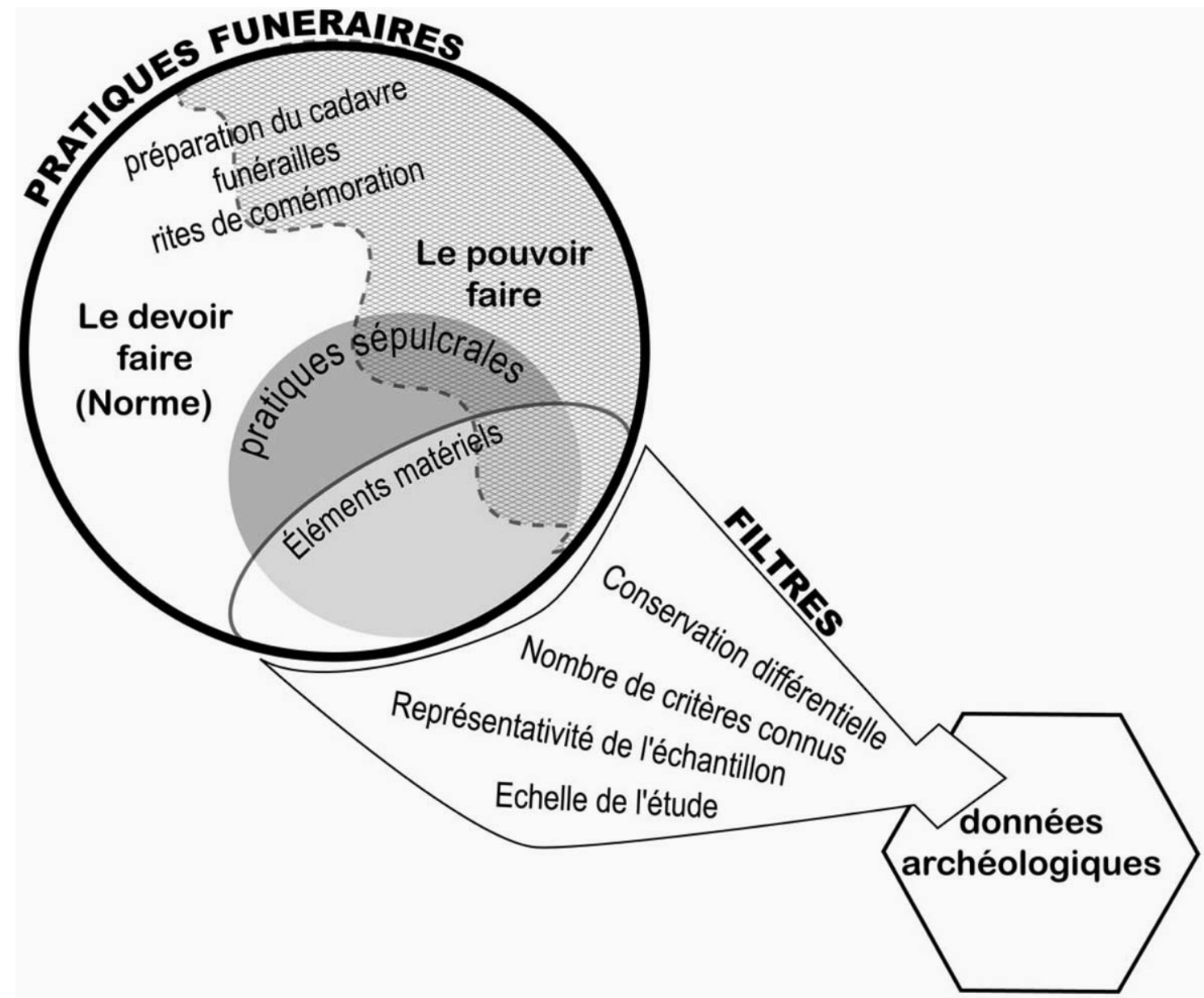

Fig. 1 Des pratiques funéraires aux données archéologiques / From funerary practices to archaeological facts

exception, se reflète directement dans toutes les tombes prises en compte. En outre, l'interdiction se traduisant comme l'obligation " de ne pas faire », la norme absolue s'exprime encore par tous les comportements qui ne sont jamais attestés (Fig. 2). La déviance, volontaire ou non, s'exprime par un écart à la norme. Une variable présente dans « pratiquement » tous les cas aura donc toutes les chances d'être également édictée par la norme.

Dans les cas où la norme est conditionnelle et s'applique donc différemment selon telle ou telle partie de la population, c'est la corrélation entre les variables qui indiquera que le geste n'est obligatoire que sous certaines conditions. Au contraire, si les variables observées semblent indépendantes et variées d'une tombe à l'autre ou d'un ensemble funéraire à l'autre, on peut penser que les gestes observés sont plutôt le fait d'un choix. La variation chronologique de tel ou tel trait peut aussi fournir une indication sur la nature des pratiques étudiées. On peut supposer que les comportements qui relèvent de l'obligation, qu'ils soient imposés ou acceptés, auront une plus grande stabilité dans le temps que ceux qui dépendent des phénomènes de mode et dont les variations seront plus rapides.

\section{Archéologie du fait funéraire}

Notre approche se voulant empirique et pragmatique, l'étude de la norme est donc fondée sur les faits archéologiques. Pour autant et concernant le monde funéraire, quels sont ces faits ? Si on considère les sépultures (cas majoritaire pour l'archéologue), ces faits concernent un squelette, un lieu, qui peut comprendre des pierres de taille diverses, et des objets.

Pour aborder la norme, nous dépassons ces données élémentaires, qui définissent grossièrement la sépulture en archéologie, et utilisons leur interprétation. Ainsi, au squelette nous préférons le défunt tel qu'il fut originellement déposé. Par ce biais nous abordons la position, l'orientation et l'apprêt du corps. Les funérailles mettant avant tout en jeu non une fosse, mais une sépulture, nous interprétons sa forme initiale, son mode de construction, ses aménagements. Pour les objets, nous recherchons leur relation au défunt ou à la sépulture. Ce sont ces premières interprétations qui font l'objet d'un traitement statistique. S'il est d'usage, dans notre discipline, de ne pas remettre en cause les faits bruts, 

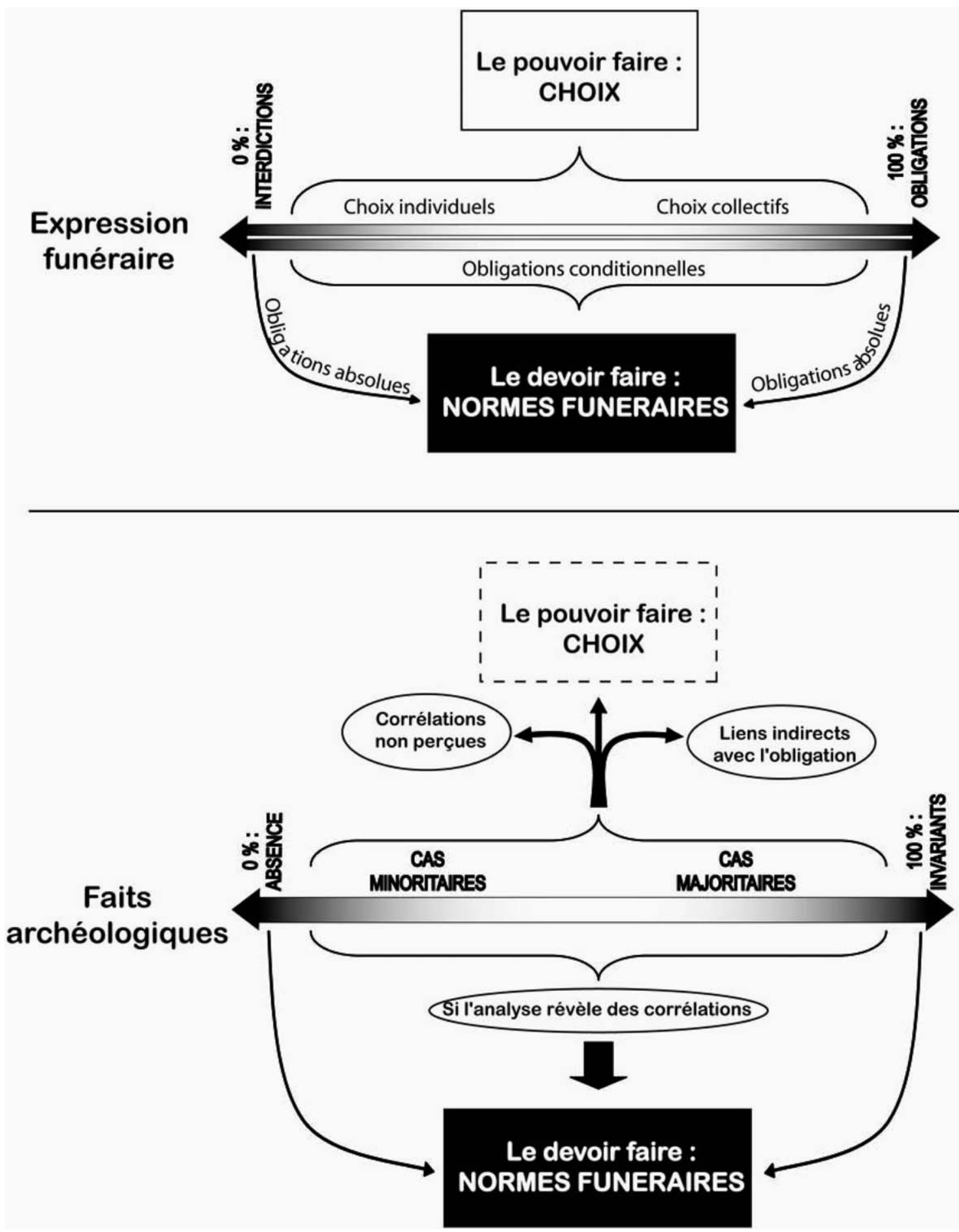

Fig. 2 Expression funéraire versus faits archéologiques. La simple observation des pratiques funéraires distingue d'un côté les choix et de l'autre les obligations. En revanche, sur le plan archéologique, seule une part des obligations est aisément caractérisable. L'impossibilité de tester toutes les corrélations comme un hypothétique lien indirect avec une obligation initiale empêchent d'affirmer qu'un geste relève d'un choix / Funerary expression versus archaeological facts. A mere observation of the funerary practices distinguishes choices from obligations. Nevertheless, with archaeological data only a portion of the obligations can be easily recognized. The impossibility of testing all correlations for a hypothetical, indirect link to an obligation prevents us from asserting that a specific practice represents a choice 
il n'est cependant pas question de ne voir dans le traitement statistique qui conduira à dégager la norme qu'une procédure mécanique : il est conditionné par notre capacité à restituer les faits matériels initiaux à partir du document archéologique.

Il ne s'agit là que d'une première difficulté. En effet, pour accéder à la norme funéraire, il nous faut résoudre, ou pour le moins prendre en compte, les problèmes de lecture liés à ces « faits matériels initiaux ». Il va de soi que l'archéologie n'a des rites funéraires qu'une image filtrée.

Le premier de ces filtres est l'échantillonnage. Tant sur le plan qualitatif que quantitatif, il nous faut comprendre comment les documents recueillis représentent la population initiale. Pour une raison ou pour une autre, ils ne représentent qu'une sélection des sépultures ; il conviendrait de s'assurer du caractère aléatoire ou non de cette sélection. Par ailleurs, cet ensemble initial ne représente lui-même qu'une partie des morts.

Le second problème est la quantification des faits. Nous travaillons statistiquement à partir de faits bruts, à peine interprétés (orientation, position de dépôt, présence/absence d'une préparation du corps, présence et nature du mobilier, architecture sépulcrale, aménagements internes, etc.). Dans la mesure où nous prenons en compte une restitution de la tombe initiale à partir des faits bruts, qu'évaluons-nous exactement : la fréquence d'un geste ou celle de sa manifestation archéologique, manifestation parfois très indirecte ? Notre raisonnement prend en compte la présence ou l'absence d'un caractère, quelquefois l'indétermination. Cependant, il est impossible de systématiquement prendre en compte cette indétermination, par exemple lorsqu'un objet déposé dans une tombe peut être dans un matériau pérenne ou putrescible.

\section{De la quantification des faits aux obligations initiales}

Le traitement statistique des données fait apparaître trois catégories de gestes. Les gestes invariants ne posent guère de problème d'interprétation en termes de norme. Ils renvoient directement à l'obligation initiale. Ainsi, si tous les sujets apparaissent en position fléchie sur le côté gauche, on peut légitimement supposer que cette position d'inhumation était imposée. Gardons-nous toutefois d'une interprétation trop directe : il peut encore s'agir d'un geste qui entretient un lien automatique avec l'obligation, sans pour autant être prescrit. Si la position étendue rime avec cercueil, par exemple, il faudra déterminer si c'est la position ou le contenant qui est imposé.

Les difficultés commencent avec les gestes récurrents, mais non systématiques (Fig. 2). L'interrogation porte autant sur la fréquence élevée que sur les cas où la tendance n'est pas respectée. Sur le plan archéologique, un problème de conservation a pu faire disparaître ce trait dans un certain nombre de cas ; sa reconnaissance peut être mauvaise, ou bien la signature archéologique n'est pas automatique. L'analyse peut se heurter à un problème d'échelle. Une mauvaise sélection de l'échantillon peut n'avoir pas permis d'identifier ce geste comme systématique. Dans un deuxième cas de figure, la corrélation d'un geste avec une caractéristique de l'échantillon peut permettre d'identifier son caractère invariant pour une fraction spécifique de la population. Nous n'avons malheureusement jamais la possibilité de tester toutes les corrélations. Pour ne citer que des exemples simples, l'âge des adultes n'est pas déterminable par les moyens actuels de l'anthropologie avec la précision qui serait nécessaire, et l'activité du défunt n'est presque jamais connue. Enfin, il faut encore s'interroger sur le lien entre l'obligation et sa manifestation archéologique, et sur le rapport entre l'obligation et le geste effectif.

Si un geste récurrent a sans doute un lien fort avec une obligation initiale, comment doit-on, en revanche, interpréter un geste minoritaire ? Toutes les difficultés précédentes restent actuelles, mais il convient d'ajouter d'autres interprétations. Le rituel des funérailles, aussi précis et complet soit-il, ne codifie pas les moindres détails : des gestes sont donc laissés au choix des officiants ${ }^{2}$, qu'ils choisissent librement ou bien dans une liste préétablie. Par ailleurs, il arrive que les officiants transgressent délibérément la norme, soit poussés par les circonstances, soit pour des raisons idéologiques. On peut encore penser que cette norme peut simplement être ignorée des officiants, totalement ou partiellement. Le trait peut finalement être relié à une obligation, mais très indirectement : le geste identifié n'est qu'une conséquence lointaine de la norme.

\section{À la recherche de la norme perdue}

Les études de cas présentées ci-dessous visent à donner, à travers des exemples archéologiques concrets, un aperçu des questions soulevées par la reconnaissance des normes en contexte préhistorique ${ }^{3}$. Les huit contextes culturels retenus donneront l'occasion de s'interroger sur les facteurs qui sous-tendent la récurrence ou l'absence de récurrence des pratiques observées. L'origine géographique et chronologique volontairement variée des ensembles évoqués nous a conduits à extraire des données inscrites dans un domaine culturel étroit les questions plus générales que chacun illustre. Cette diversité offre l'avantage de nous placer au-delà des grands systèmes qui peuvent régir une aire spécifique.

\footnotetext{
${ }^{2}$ Le terme officiant est ici employé sans connotation religieuse : celui qui officie.

${ }^{3}$ Les cas d'études présentés ci-après sont le résultat de recherches individuelles menées, par ordre d'intervention, par J. Leclerc, P. Chambon, G. Pereira, C. Thevenet, F. Bocquentin, J.-G. Pariat, I. Le Goff et F. Valentin.
} 
Définir un objet d'étude : les allées sépulcrales Seine-Oise-Marne

Pour une recherche sur la norme, comme pour toute recherche, il est indispensable de commencer par définir sans ambiguilté l'objet d'étude. La norme qu'on veut faire apparaître, c'est celle d'un peuple particulier - c'est-à-dire d'un corpus de sites - différent de tous les autres. Son interprétation en termes culturels sera celle de ce peuple particulier; elle pourra aussi, dans certains cas, aider à le définir.

Les sépultures collectives du Bassin parisien semblent présenter pour cela une situation archéologique éminemment favorable : des documents funéraires monumentaux, bien visibles, abondants, et dont les modes de construction et d'utilisation paraissent assez apparentés pour qu'on les ait depuis longtemps attribués sans hésitation à un même groupe humain. Une bonne occasion pour recenser les pratiques qui paraissent réellement obligatoires, et chercher en quoi elles peuvent faire système.

Cependant, la délimitation de notre objet d'étude s'avère d'autant plus délicate que les sites que nous prenons en charge ont été rattachés depuis longtemps à une très vaste et très envahissante entité culturelle, la « culture de SeineOise-Marne » [7], une culture définie pour l'essentiel par des observations en milieu funéraire. Cette ambiguité est gênante ; il fallait partir sur de nouvelles bases.

Pour définir un ensemble funéraire cohérent, choisir sa taille, et tracer ses limites, trois méthodes sont théoriquement possibles. On peut tracer des limites spatiotemporelles arbitraires (limites de siècles, limites politiques actuelles, accidents géographiques - par exemple, entre deux fleuves) : c'est la méthode la plus sûre, mais la moins intéressante. On peut aussi s'attacher à une culture définie par ailleurs sur des bases non funéraires - ce qui est s'interdire par construction d'observer une quelconque discordance entre la présence d'une culture archéologique et l'aire de diffusion d'une norme funéraire. On peut enfin vouloir éprouver l'hypothèse du caractère supraculturel des comportements funéraires, et pour cela se lancer dans la recherche autonome d'une culture funéraire. C'est ce dernier chemin qui a été suivi, en cherchant si les caractères communs observés dans les sites dont nous disposions pouvaient être associés ou dissociés de façon à dessiner sur la carte un ensemble homogène.

Des caractères communs, on en trouve assurément... mais il est rare qu'ils soient rigoureusement discriminants. Le plus remarquable, l'inhumation collective des défunts concerne la plus grande partie de l'Europe de l'Ouest et du Nord ; elle s'observe aussi au Moyen-Orient — qu'on éliminera au nom de la discontinuité géographique. On peut noter que le phénomène prend successivement deux formes différentes, ce qui justifie qu'on limite l'étude à la première, qui occupe les derniers siècles du quatrième millénaire. Autre trait remarquable, le caractère enterré du sépulcre touche lui aussi une vaste aire ouest-européenne, dont il ne permet d'exclure que les régions les plus occidentales. Les mêmes régions seront également écartées par la prise en compte d'autres pratiques caractéristiques, les procédures de condamnation, qui présentent dans ces régions occidentales un caractère spectaculaire qui s'oppose clairement à la discrétion recherchée ailleurs. Enfin, la disposition et le rôle du mobilier funéraire semblent bien être communs à l'ensemble de l'aire qui nous intéresse, mais couvrent une aire trop étendue pour être réellement discriminants. Encore un critère d'utilisation difficile.

Finalement, c'est l'attachement au modèle de l'allée sépulcrale qui constitue sans doute le critère le plus caractéristique. Ces monuments suivent strictement un modèle d'architecture très stéréotypé, qui ne peut que traduire de fortes contraintes fonctionnelles. Associés à des règles communes pour la position des corps, ils constituent vraiment le meilleur indice d'une norme funéraire particulière.

Il est vrai que ce critère, pour sûr qu'il soit, n'est pas tout à fait discriminant, parce qu'il inclut les sites d'Allemagne du Nord (en particulier de Westphalie) et les sites de FrancheComté. Ce n'est pas un réel problème : la discontinuité géographique donne opportunément une bonne raison d'éliminer ces sites. Il reste à écarter (peut-être provisoirement) les territoires les plus occidentaux du Bassin parisien, qui semblent présenter quelques traits un peu particuliers, pour voir s'offrir comme objet d'étude une norme funéraire homogène sur un territoire bien délimité : le Bassin parisien central (Nord, Picardie, Île-de-France, et parties adjacentes de la Bourgogne et de la Champagne).

Cet ensemble a été constitué par élimination en considérant uniquement les données funéraires, sans jamais prendre en compte les limites culturelles tracées par les néolithiciens. Cependant, dans le même temps, ces derniers ont fait éclater l'ensemble traditionnel « Seine-Oise-Marne », en n'en laissant qu'un ensemble bien restreint dans le temps [8] et dans l'espace [9]. Ils ont ainsi défini un nouvel objet d'étude qui recoupe plus ou moins celui que nous avons défini : cherchant une norme funéraire autonome, aurions-nous seulement retrouvé des limites culturelles?

\section{Tracer les limites de la norme en procédant trait par trait : le bassin Seine-Yonne entre 4600 et 4200 av. J.-C.}

Comme nous venons de l'évoquer, pour reconnaître la norme funéraire, il convient au préalable de définir les contours de l'ensemble à étudier, en d'autres termes la population concernée. Malheureusement, en préhistoire, nous n'avons pas directement accès à la population : la géographie et la chronologie des groupes humains sont fondées sur les constructions que nous appelons «cultures », qui se résument bien souvent, pour la période néolithique, à des styles céramiques. Il n'est guère étonnant que dans les contextes de 
fortes densités d'entités type « cultures », les pratiques funéraires ne paraissent pas s'accorder avec un tel découpage.

Pour définir notre objet d'étude, il nous reste le document lui-même, avec le risque de raisonnement circulaire. Pour limiter la difficulté, nous utilisons les faits archéologiques bruts, ou à peine interprétés. Nous examinons leur cohérence spatiale et temporelle avant d'aller plus loin et de tenter une analyse du système funéraire. Cependant, cette cohérence nous échappe bien souvent et les critères utilisés pour définir les ensembles dessinent des limites qui ne se superposent pas.

Notre exemple est ici constitué par les pratiques funéraires du bassin Seine-Yonne dans la première partie du Néolithique moyen (4600-4200 av. J.-C.). Le corpus est important pour cette période, près de 170 tombes, avec une documentation récente. L'argument initial, pour regrouper les huit ensembles pris en compte (Balloy, Chichery, «Sur les Pâtureaux », Gron, Monéteau, Orville, Passy, Vignely « Porte aux Bergers » et « Noue Fenard »), outre la concentration dans le temps et l'espace, est principalement lié à la position d'inhumation, étendue sur le dos. Au sein du Néolithique français, cette position est exceptionnelle ; elle accompagne un type de sépulture original : le type «Balloy», soit un caveau aménagé dans une fosse pour accueillir un défunt inscrit au sein d'un contenant mobile rigide [10]. Nous avons donc réuni les nécropoles contemporaines présentant des inhumations en position étendue sur le dos, que ces inhumations soient ou non exclusives au sein de ces nécropoles.

Pour tous les cimetières retenus, nous avons recherché les principaux caractères : les éléments structurant l'espace funéraire, l'agencement des sépultures, l'architecture sépulcrale, le mode d'inhumation, la position du cadavre, son orientation, le mobilier qui l'accompagne et son mode d'association. Sur chacun de ces plans, toutes les sépultures et toutes les nécropoles n'observent pas les mêmes règles, loin s'en faut.

Le nombre d'individus inhumés est très variable, certains ensembles ne s'inscrivant qu'à la marge dans la définition de la nécropole. Les tombes sont parfois incluses dans des monuments, mais pas systématiquement. Les positions d'inhumation opposent sujets fléchis et sujets étendus sur le dos. Par exemple, l'orientation « tête à l'est » est plébiscitée à Balloy, mais à Passy la majorité des sépultures ont une orientation inverse, et à Vignely « Porte aux Bergers » on ne décèle aucune préférence marquée. Le sexe des inhumés structure plusieurs ensembles, comme Balloy ou Gron, mais pas tous.

Si l'on perçoit confusément que deux nécropoles voisines témoignent, malgré de légères dissemblances, d'une même entité funéraire, le problème devient plus épineux lorsqu'on élargit l'échelle. En effet, pour regrouper deux ensembles, il suffit de considérer que telle option, qui semblait essentielle à l'échelle de la nécropole $\mathrm{x}$, ne correspondait qu'à un choix local, sans signification profonde du point de vue de la norme funéraire : c'est ainsi le cas de l'orientation tête à l'est systématique à Passy, qui n'est plus la règle à Balloy $[11,12]$. Cependant, pour procéder à des regroupements plus importants, nous devons chaque fois relativiser d'autres critères. Finalement, aucun critère n'est commun à la totalité des sépultures prises en compte, ni ne leur appartient en propre.

Si les critères pris en compte ne permettent pas de définir un ensemble cohérent, il faut se résoudre à une alternative : soit l'ensemble que nous avions créé a priori est de fait incohérent, et comprend des manifestations qui renvoient à différents systèmes funéraires, soit les critères sélectionnés ne traduisent pas directement la norme, et ne permettent donc pas d'en tracer les contours. Que reste-t-il comme solution? Dans ce cas précis, une démarche inverse peut être envisagée : ébaucher une interprétation du système, à partir d'un gisement suffisamment complexe ou riche pour en offrir l'occasion, et distinguer, dans le reste du corpus les témoignages qui peuvent s'inscrire dans ce système de ceux qui ne le peuvent pas. C'est ici traquer l'idée derrière le geste.

\section{Deux façons de concevoir la norme funéraire : les hautes terres du Michoacán}

Nous comparons ici des ensembles funéraires contemporains (600-900 ap. J.-C.) issus de deux secteurs contigus du centre-ouest mexicain : les hautes terres du Michoacán [13] qui s'étendent au sud du fleuve Lerma et le massif de Barajas (sud-ouest du Guanajuato) au nord de celui-ci [14].

L'intérêt de notre comparaison tient au fait que les sociétés issues de ces deux régions donnent à voir des pratiques funéraires radicalement différentes alors que, dans d'autres domaines de la culture matérielle, elles présentent de nombreuses affinités. Les sépultures mises au jour sur les sites du massif de Barajas témoignent d'une forte homogénéité qui contraste notablement avec le caractère hétérogène des pratiques répertoriées au Michoacán. Dans un cas, on est donc tenté de voir la marque de règles assez strictes tandis que dans l'autre, la diversité des pratiques laisse planer un doute sur le caractère normé de ces dernières. Comme nous allons tenter de le montrer, ces différences correspondent plutôt à une conception différente de la norme plutôt qu'à la présence ou l'absence de celle-ci.

Commençons par examiner les pratiques sépulcrales des sites du Massif de Barajas. Les données dont nous disposons montrent que les défunts étaient inhumés dans de petits cimetières localisés à l'intérieur de bâtiments à vocation funéraire localisés à proximité immédiate des habitats. Lorsque les ensembles ont été fouillés de façon extensive, on constate qu'ils regroupent chacun 30 à 40 sujets qui ont été enterrés successivement dans un espace assez réduit. Le 
creusement répété des fosses dans un secteur exigu où chaque tombe n'était apparemment pas nettement individualisée a provoqué, au final, de nombreuses perturbations. Le « bruit » provoqué par ces perturbations mises à part, on constate que les pratiques sépulcrales s'effectuent invariablement selon les mêmes modalités : il s'agit de sépultures primaires dans lesquelles le corps est placé en position allongée, les membres inférieurs en extension; l'axe du corps suit une direction est-ouest, la tête étant toujours du côté est. Le contenant employé semble n'être prévu que pour recevoir un seul sujet : il s'agit d'une fosse oblongue à peine plus grande que le corps. Quelques indices taphonomiques permettent de supposer que le cadavre était placé dans une enveloppe semirigide en matériaux périssables. Le mobilier d'accompagnement est constitué presque exclusivement de récipients céramiques que l'on trouve en nombre variable d'un dépôt à l'autre. En revanche, les autres catégories d'objets (outils, parure) sont rares.

Si l'on compare, à présent, les pratiques des habitants du secteur de Barajas à celles de leurs contemporains du Michoacán, on est frappé par les divergences quasi systématiques entre ces deux zones. À la relative monotonie des comportements funéraires décrits précédemment succède un foisonnement qui dénote d'abord d'une nette « individualisation $» d u$ traitement funéraire. Notons que ces variations sont perceptibles d'un site à l'autre, mais également à l'échelle intrasite. Si l'inhumation est là aussi de règle, en revanche, la position (étendu, fléchi sur le côté, le dos ou le ventre, assis) et l'orientation du corps sont très diverses. À la différence de ce que nous observons à Barajas, l'inhumation est clairement suivie de nombreuses manipulations (prélèvements, transferts, inhumations secondaires) des ossements qui interviennent après la décomposition ou plus exceptionnellement (comme sur le site de Tingambato), après une phase de décharnement actif. Le mobilier funéraire est là aussi largement plus varié et inclut une gamme assez large d'objets et de matières premières.

Mais il serait trompeur de limiter notre comparaison à une simple opposition entre pratiques homogènes (= normées) d'un côté (Barajas) et pratiques hétérogènes (peu ou pas normées) de l'autre (Michoacán). En effet, parmi la variété des gestes funéraires observés au Michoacán, on perçoit un certain nombre de régularités qui évoquent l'existence de normes partagées. Celles-ci se fondent, semble-t-il, sur un principe d'individualisation et de différentiation. L'architecture et l'organisation des ensembles funéraires en témoignent. Là, les inhumations ont été pratiquées dans un espace balisé qui garantit l'intégrité de chaque dépôt. Ces derniers peuvent contenir un nombre variable de personnes (tombes individuelles, doubles, collectives), mais l'architecture est en adéquation avec le fonctionnement de la tombe : les sépultures collectives sont pratiquées dans des chambres souterraines en pierre munies d'un système d'accès ; les sépultures individuelles ou doubles sont placées dans des contenants diversement élaborés (coffres, fosse en niche, fosse simple, urne).

Mais c'est la corrélation entre les types de contenants, le mobilier et le sexe du défunt [15] qui montre que les variations observées ne sont pas aléatoires, mais qu'elles sont structurées en fonction de normes. Elles reflètent clairement un traitement inégal face à la mort. Les sépultures féminines sont plutôt associées aux contenants les plus simples et à un mobilier utilitaire d'origine locale. En revanche, certains hommes et quelques sujets immatures (masculins ?) ont nettement fait l'objet d'un traitement favorable perceptible dans l'architecture de leur tombe et la richesse du mobilier. Ces personnages ont pour point commun leur association avec des objets qui renvoient à la guerre (armes, parures symbolisant le statut guerrier) et aux pratiques religieuses (encensoirs, instruments de musique, pigments).

Pour conclure, nous insisterons sur le fait que les deux corpus analysés témoignent de pratiques qui, dans les deux cas, constituent des systèmes cohérents, mais qui obéissent chacun à des principes distincts. Dans le cas du Michoacán, malgré l'hétérogénéité dominante, les importantes variations que nous observons entre les défunts témoignent d'un système de différentiation codifié fortement connoté socialement. Dans le cas de Barajas, les pratiques funéraires font preuve, au contraire, d'une grande homogénéité. Les normes s'expriment par des invariants et elles offrent une image plus homogène du monde des morts.

\section{Des normes funéraires comme vecteurs d'identité et d'altérité : le Rubané du Bassin parisien}

Que peuvent donner à voir de la société qui les a produites les pratiques funéraires d'un groupe humain, quand celui-ci s'insère au sein d'un complexe culturel aussi vaste géographiquement que long chronologiquement ? C'est à cette question que peut se résumer la tentative d'appréhender la norme funéraire durant le Rubané (Céramique Linéaire) du Bassin parisien. Cette tentative se heurte toutefois à un premier truisme. Si l'on peut mettre en évidence un caractère normé de la pratique funéraire, celui-ci ne peut concerner qu'une partie de la population « inhumante » [16] : peu de sépultures sont connues pour une aire géographique vaste et une durée longue, ces dernières témoignant par ailleurs d'un recrutement funéraire ciblé [17]. C'est donc seulement une norme funéraire au sein du Rubané du Bassin parisien qui se trouve à notre portée, norme concernant des sépultures à inhumation toujours primaire et toujours localisées au sein des villages rubanés. Celles-ci constituent-elles pour autant un ensemble homogène ? En d'autres termes, si certains 
gestes funéraires s'avèrent variés, cette variation s'inscritelle tout de même dans un système ?

La culture rubanée, première société agropastorale d'Europe continentale, constitue ainsi l'un des plus vastes complexes culturels qui se soit développé, durant la première moitié du sixième millénaire avant notre ère [18]. En son sein, le Rubané du Bassin parisien (« Rubané moyen/récent champenois » et "Rubané Récent du Bassin parisien », $5200-4900$ av. n. è.) compose une entité géographique et temporelle clairement circonscrite qui réunit relativement peu de sépultures : nous en avons retenu 124 sur près de 170 connues, selon la fiabilité des attributions chronologiques et la qualité de la documentation.

Les sépultures rubanées du Bassin parisien présentent de nombreux points communs : leur localisation est limitée aux habitats, leur structuration est stéréotypée, il s'agit exclusivement d'inhumations primaires, les défunts y sont toujours déposés la tête dirigée vers l'orient et les membres inférieurs fléchis. Autant de gestes invariants que l'on est tenté d'interpréter en termes d'obligation au sein de ce corpus. Face à cela, de nombreux gestes sont variables, mais leur degré de variabilité diffère. Certains constituent une écrasante majorité (par exemple, $91 \%$ des sujets ont les membres inférieurs fléchis vers la gauche), d'autres peuvent sembler statistiquement aléatoires $(50,8 \%$ des sépultures contiennent des objets) tandis que quelques-uns sont anecdotiques (objets présents en très peu d'exemplaires, manipulations postmortem, etc.). Autant de gestes dont la représentativité peut se prêter à de multiples interprétations : geste normé, mais pas toujours reconnu, geste normé réservé à certains seulement, écart à la norme, geste non normé, etc., la liste ne se veut pas exhaustive. Au-delà du concept de norme funéraire, ces différents gestes et leur représentativité également différente permettent d'appréhender divers niveaux d'intégration au sein du Bassin parisien. On peut alors opposer des gestes invariants et observables dans l'ensemble de cette aire géographique, à des gestes - apparaissant majoritaires, minoritaires ou anecdotiques - observables dans certaines régions exclusivement, caractérisant certains sites par rapport à d'autres, propres à quelques individus seulement ou encore caractéristiques d'une étape chronologique. Ainsi se dégageraient à partir des divers gestes funéraires, l'intégration à la société rubanée du Bassin parisien, l'intégration aux différents groupes régionaux qui la composent, l'intégration aux différents villages qui constituent ces groupes ; enfin, à l'intérieur de chacun de ces sous-ensembles, place serait tout de même laissée à l'expression des individualités, ou simplement des sentiments portés au défunt ou encore d'événements particuliers.

Les normes funéraires rubanées du Bassin parisien formeraient alors effectivement un système, système vecteur d'identité et d'altérité à l'intérieur de cette aire géographique. Ellemême marquerait son autonomie par rapport au reste de la
Céramique Linéaire, tout en affirmant son appartenance à ce vaste ensemble culturel. Ainsi, si les nécropoles sont absentes dans le Bassin parisien contrairement à d'autres régions de cette culture, l'organisation des sépultures dans les habitats reproduit néanmoins un système binaire d'implantation des tombes tel qu'il peut se dessiner ailleurs dans cette culture où coexistent alors nécropoles et sépultures en habitat (Thevenet, travaux de doctorat en cours).

\section{Écarts à la norme en contexte culturel dominant : les Natoufiens du Proche-Orient}

Au sein d'un ensemble culturel dominant, on s'interroge sur la marge de manœuvre dont disposent les différents groupes pour imposer leurs propres règles chez eux. Déroger à la norme funéraire dominante se fait-il par des pratiques masquées, ostentatoires, de façon anarchique ou canalisée ? Nous aborderons cette question à travers les communautés épipaléolithiques natoufiennes du Proche-Orient (13000 10000 av. J.-C.).

L'entité culturelle natoufienne est définie sur la base d'une évolution chronologique (basée essentiellement à l'origine sur l'industrie lithique, mais confortée par le reste de la culture matérielle et les datations), d'un mode de vie (des sédentaires ou en cours de sédentarisation), de techniques (les industries, chasse, architecture), et de référents symboliques (motifs décoratifs, associations d'objets) communs [19]. Toutefois, quel que soit le domaine d'étude, des particularités locales sont observées. Les pratiques funéraires s'inscrivent-elles dans cette dualité et de quelle façon ? Une fois les contours d'une norme dominante explorés, on s'interroge sur les écarts qui sont faits à cette norme.

À travers les récurrences, les invariants ou, au contraire, les cas exceptionnels du corpus disponible ${ }^{4}$ (environ 400 individus), il nous semble possible de déceler différentes expressions de pratiques normées. On observe, en premier lieu, des pratiques plus fréquentes que d'autres, voire majoritaires, qui semblent répondre à des valeurs collectives. Cela concerne par exemple le mode d'inhumation, préférentiellement primaire individuel (46\%) en fosse simple, mais qui cède la place à une majorité de sépultures plurielles $(78 \%)$ le temps du Natoufien récent (évolution commune des différents sites). En second lieu, on note des constantes ou des absences remarquables que l'on est tenté de ramener à un système codifié plus strict de type interdiction/obligation. Par exemple, lors de dépôts simultanés, les enfants décédés avant cinq ans sont associés exclusivement à des hommes.

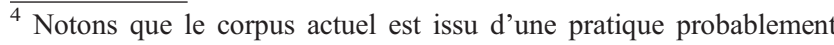
minoritaire, celle d'enterrer les morts à proximité des maisons. Elle est cantonnée aux seules occupations pérennes de la région centrale du territoire natoufien (vallée du Jourdain ; Galilée/Carmel).
} 
La position d'inhumation qui paraît la plus naturelle, étendue sur le dos, est strictement limitée aux inhumations en grotte et n'est pratiquée qu'au début du Natoufien. Les pendeloques associées aux défunts sont limitées à certaines catégories et ne sont présentes qu'au Natoufien ancien; les dépôts de faune ne concernent que certaines parties anatomiques [19] et disparaissent au Natoufien final.

À quoi renvoie cette apparente distinction entre préférences et obligations ? À des systèmes funéraires indépendants qui coexistent en interférant peu ou pas l'un sur l'autre ; ou bien à un système unique basé sur des règles plus ou moins rigoureuses, hiérarchisées. On peut envisager qu'on est tout simplement en présence d'une norme unique basée sur des règles identiques aujourd'hui perçue partiellement soit que la rigueur de la norme ne soit plus décelable à travers les trois millénaires qui la distendent, soit, au contraire que les règles considérées comme strictes ne reflètent que l'état d'avancement des fouilles et devront souffrir de plus en plus d'exceptions à l'avenir.

À présent que ce cadre des pratiques funéraires normées est posé, faut-il interpréter les particularités locales comme des transgressions de ces règles collectives ? Ces particularités peuvent être classées en deux catégories : celles, d'une part, qui marquent une rupture par rapport à ce qui est observé partout ailleurs. Ainsi, alors que les fosses sépulcrales simples sont la règle, les tombes du site d'Hayonim sont, en majorité, des structures de pierre élaborées ; et, à Mallaha, certaines fosses sépulcrales sont chaulées. Il y a donc bien écart à la norme, mais il se trouve que ces structures particulières sont associées à des pratiques rares : funérailles en plusieurs temps à Hayonim et dépôts successifs à Mallaha. $\mathrm{Ne}$ serait-on pas dans le cas de pratiques minoritaires normées ? La seconde catégorie fait référence à certaines particularités locales qui semblent dériver d'une entente collective préalable sur la façon d'exprimer une identité locale. C'est peut-être le cas du recrutement funéraire sélectif sur des critères liés à l'âge et au sexe selon des quotas qui varient (mais on est fortement dépendant de la représentativité différentielle des échantillons dans cet exemple). C'est certainement le cas pour le choix des éléments de parure spécifiques à un groupe, de même que leur nombre et la façon de les porter. Il semble que l'on touche ici à la structuration sociale et identitaire du groupe qui ne rompt pas, mais transcende les prescriptions collectives.

Les pratiques funéraires natoufiennes ont longtemps été considérées comme dépourvues de normes (pour une synthèse sur le sujet [20]). C'est sans doute la diversité des positions d'inhumation qui a masqué une réalité plus complexe. Une étude synthétique fait apparaître des récurrences, des corrélations répétitives : on a affaire à des pratiques, si ce n'est prescrit du moins, conventionnelles. Quant aux « écarts » observés, ils apparaissent finalement circonscrits dans des cadres préalablement définis. Que reste-t-il des pratiques non normées ? Certes, les positions d'inhumation sont individualisées, mais le soin apporté à celles-ci évoque le respect d'une ordonnance, d'une norme qui ne se matérialise pas par un fait archéologique standardisé. Comment, alors, distinguer la règle de la pratique exempte de toutes contraintes extérieures ?

\section{Représentativité des données archéologiques et pratiques funéraires : le Bassin parisien entre 4200 et 3400 av. J.-C.}

La faiblesse numérique d'un corpus de tombes et son hétérogénéité, qu'elles reflètent une réalité historique ou un état de la recherche, incitent généralement à conclure à une absence d'unité de pratique. Cette opinion est-elle justifiée, ou peuton rechercher une ou des normes au sein même de cette diversité et en dépit d'un faible effectif?

Du point de vue historique, certains traitements sont appliqués à une majorité de la population, tandis que d'autres témoignent d'un comportement minoritaire ou marginal. Il doit être possible de faire ressortir des récurrences en soumettant un corpus, aussi diversifié soit-il, au filtre de plusieurs critères. Dès lors, comment interpréter d'éventuelles redondances ? Les données archéologiques permettent-elles de déterminer le caractère majoritaire ou minoritaire d'une pratique au sein d'un groupe?

Le cas des pratiques funéraires du Bassin parisien au début du Néolithique moyen II illustre ces questionnements tout en interrogeant justement la nature du lien entre traces archéologiques et réalité historique.

La période citée couvre près d'un millénaire entre $4200 / 4100$ et $3400 / 3300$ av. J.-C. Durant une première phase, située entre 4200/4100 et 3800 av. J.-C., trois ensembles culturels sont représentés par des corpus céramiques provenant de divers sites : le Chasséen septentrional à l'ouest, le Michelsberg au nord et à l'est, et le groupe de Noyen à l'est. Dans chacun de ces horizons culturels, des restes humains issus de fossés d'enceinte ou de zones d'épandage, des tombes isolées ou des squelettes dans des puits de mine ont été retrouvés. Mais tous ces vestiges ne relèvent pas nécessairement du domaine funéraire, certains ayant pu se retrouver par hasard sur le lieu d'enfouissement ultime [21]. Dans le cadre présent, nous avons choisi de travailler uniquement à partir des tombes, seuls témoins incontestables d'une pratique funéraire.

D'un point de vue statistique, ces sépultures sont très peu nombreuses, environ 50 sur une période de 400 ans. Cette faible représentation peut être interprétée de deux façons. Un maigre effectif de tombes peut résulter d'une pratique minoritaire ou marginale, traduisant une réalité historique. Il peut aussi s'agir d'une sous-représentation d'un traitement majoritaire, passé inaperçu du fait de l'érosion des sites ou de 
territoires partiellement explorés faute de fouilles extensives (fonds de vallée privilégiés au détriment des plateaux).

Malgré ce déficit quantitatif, un classement des données est-il possible en vue de corréler certains faits ? Des tris sélectifs réalisés parmi les situations rencontrées ont en effet permis de dégager des récurrences. Seules les tombes datées avec fiabilité, soit par le mobilier soit par $\mathrm{C}^{14}$, ont été prises en compte et cinq grandes familles de critères (contexte d'apparition du squelette, mode d'inhumation, nature du dépôt, recrutement, mobilier) ont été utilisées pour effectuer des tris. Ces différents filtres ont conduit à observer des répétitions parmi les 20 tombes du corpus. Ainsi, dans $80 \%$ des cas, on constate qu'une fosse a été creusée pour y déposer un ou plusieurs corps, l'inhumation individuelle dominant largement. Par ailleurs, le sujet repose généralement en position repliée sur le côté gauche $(60 \%$ des cas). Les autres critères testés ont fait ressortir des variations importantes qui semblent indiquer que la position du mobilier par rapport au squelette ou l'âge du défunt, par exemple, ne sont pas des éléments déterminants du rite.

Finalement, l'inhumation en fosse d'un sujet replié sur le côté gauche est la situation la plus courante, sans toutefois être exclusive. Cette récurrence combine seulement trois des 15 critères testés, laissant place à une forte variabilité (âge, sexe, position du mobilier, etc.). Une absence volontaire de rigidité dans la norme imposée est donc à envisager. Nous serions en présence d'une pratique funéraire relativement flexible et peu unifiée. On peut ainsi envisager le cas d'une pratique normée laissant place à de nombreuses déclinaisons, où chaque groupe avait la responsabilité des enterrements des siens et du culte funéraire qui les concernait.

Bien qu'il soit possible d'entrevoir des corrélations, il reste complexe d'établir un lien entre ces données archéologiques et la réalité historique, donc de déterminer avec certitude le caractère minoritaire ou majoritaire d'une pratique en présence d'un corpus aussi faible. Cela n'implique pas qu'un ensemble plus important numériquement doive d'emblée être considéré comme significatif : les corpus de tombes préhistoriques, si importants qu'ils soient, sont toujours bien en deçà des estimations démographiques les plus minimales. La nécessaire remise en question de la représentativité doit être au cœur de chaque nouveau cas analysé.

\section{Comprendre les transformations de la norme funéraire : son interaction avec d'autres dimensions d'une culture : le site protohistorique d'Ifs}

L'angle choisi pour notre intervention est celui de la détection, en archéologie, des transformations de la norme funéraire. L'attention est portée sur le déclenchement d'actions funéraires nouvelles, que ces dernières soient perçues comme complexes, car concernant une séquence de gestes ou comme simples, car portant sur un seul trait.
Le site protohistorique d'Ifs Object'ifs Sud (Calvados) fouillé dans le cadre de l'Inrap offre, avec plus de quatre siècles d'occupation funéraire continue, la profondeur temporelle nécessaire pour percevoir la mise en place de changements [22]. Par ailleurs, l'importance des surfaces décapées sur près de 50 ha ouvre une fenêtre sur l'ensemble de l'organisation du tissu rural. Elle a révélé deux habitats distants seulement de $200 \mathrm{~m}$ et 16 ensembles funéraires étroitement imbriqués dans les espaces domestiques. De la sorte, il est possible de questionner les mécanismes des changements d'un point de vue assez large pour intégrer les interactions entre le domaine funéraire et d'autres dimensions d'une culture (politico-religieuse ou sociale) et proposer de possibles schémas d'actions $[23,24]$. Sur ce site, les normes funéraires se modifient en profondeur à deux moments.

Ainsi, la posture donnée au corps à la fin $\mathrm{du} \mathrm{VI}^{\mathrm{e}}$ siècle change au cours du $\mathrm{V}^{\mathrm{e}}$ siècle avant J.-C. En considérant une série de quatre traits (posture, orientation du corps et de la tête, traitement du cadavre), la situation au $\mathrm{VI}^{\mathrm{e}}$ siècle montre des actions reproduites à l'identique à chacune des funérailles (environ 40 sujets). Les corps sont placés sur le dos ou le côté, avec les genoux fléchis, la tombe est orientée selon un axe SO/NE, la tête est placée au SO sauf pour les enfants en situation inversée et pour quelques tombes adventices placées dans le fossé d'enclos. Leur orientation dépend alors des branches du fossé qui les accueille. Une modification des normes s'opère au cours $d u V^{e}$ siècle qui conduit à la variation des actions possibles (corps étendu, orientations diverses de la tombe et de la tête).

Ces changements sont liés à des changements dans le domaine politico-religieux exprimé par la structuration de l'espace. À la fin du $\mathrm{VI}^{\mathrm{e}}$ siècle coexistent quelques petits ensembles de tombes (deux tombes) avec une autre de dimension communautaire (29 tombes) structurée autour d'un enclos quadrangulaire. Les ensembles funéraires s'intègrent dans un espace matérialisé par des enclos fossoyés accolés qui préfigurent la mise en place d'un paysage agraire, organisé selon des éléments rectilignes et quadrangulaires. À la fin du premier âge du Fer, l'organisation de l'espace rural se poursuit, les habitations se mettent en place. Quant aux morts, le regroupement en nécropole disparaît au profit d'un système qui intègre de petits groupes de tombes épars dans le paysage agraire, mais reliés par une même logique d'implantation le long des fossés parcellaires, des clôtures d'habitats ou au débouché d'un chemin qui conduit à une habitation.

Il est fait ici l'hypothèse d'interactions entre la dimension funéraire reflétée par la posture du corps et le domaine politico-religieux perçu par l'organisation de l'espace. On note la forte normalisation de l'orientation de chacune des composantes, et ce, à différentes échelles : une même orientation est donnée aux corps, à la tombe, aux rangées de 
tombes, aux branches de l'enclos funéraire et enfin aux enclos du parcellaire. Au cours du $V^{e}$ siècle, le rapport à l'espace se modifie. Les groupes de tombes s'implantent dans les habitats et la forte convergence entre la structuration de l'espace et la posture du corps se perd. Corps, tombes, rangées de tombes ne s'alignent plus sur les orientations des fossés parcellaires et des chemins.

Le III ${ }^{\mathrm{e}}$ siècle voit l'introduction d'un nouveau traitement du corps, la crémation, sans changement de l'organisation des espaces funéraires, puisque à côté des sépultures à inhumation primaire sont implantées les tombes à crémation. La dimension sociale se manifeste par la présence d'un bracelet annulaire en bronze, parfois associé à un bracelet en lignite. On le rencontre souvent associé à des torques dans les tombes à inhumation dites riches à la fin du premier âge du Fer et à La Tène ancienne. $\mathrm{Au} \mathrm{II}^{\mathrm{e}}$ siècle alors que la norme est l'absence d'objet ou le port de fibule en fer, se retrouve, une seule fois, dans une tombe dite riche, l'association bracelets de bronze et en lignite. La présence de ces objets inscrits dans une tradition signerait la transmission d'une mémoire sociale et suggèrerait que des enjeux sociaux leur sont toujours attachés. Ce schéma d'action est réactivé au $\mathrm{II}^{\mathrm{e}}$ siècle dans un contexte particulier, celui d'une crémation, pratique nouvelle à La Tène moyenne.

Un des intérêts de cette situation est de montrer l'activation de schémas d'actions traditionnels liés aux normes sociales alors que se mettent en place, dans une sorte de continuité, de nouvelles normes funéraires relatives au corps.

\section{Influence d'un changement historique sur la norme funéraire : les Sakalava de Madagascar}

Quelle peut être l'influence d'un changement historique sur la norme funéraire ? La question est abordée ici par le moyen d'une comparaison entre modes funéraires anciens et actuels des groupes Sakalava installés dans la presqu'île de Narinda (côte nord-ouest de Madagascar) depuis le XVIII ${ }^{\mathrm{e}}$ siècle. Les premiers sont documentés par des observations archéologiques réalisées dans cinq abris sépulcraux et les seconds par des observations ethnographiques [25,26]. Ces groupes connurent d'importants changements culturels et sociopolitiques aux $\mathrm{XIX}^{\mathrm{e}}$ et $\mathrm{XX}^{\mathrm{e}}$ siècles qui aboutirent à une restructuration profonde de leur système identitaire : contact affirmé avec des populations européennes et colonisation, effondrement du système sociopolitique lié à des conflits interethniques, et accession à l'indépendance. L'étude montre que les restructurations consécutives qui ont favorisé l'abandon de certaines traditions anciennes n'ont que peu affecté les pratiques funéraires caractérisées aux deux périodes par une normalisation stricte du traitement des corps et de la gestion de l'espace sépulcral.
En effet, au-delà de variations propres à chaque mode funéraire, plusieurs aspects des pratiques observées dans les abris sépulcraux trouvent des parallèles manifestes dans les descriptions ethnographiques. Traitement des défunts et gestion de l'espace sépulcral ancien et actuel ont en commun : un enveloppement du corps dans des étoffes, une inhumation primaire et définitive sur le côté variant selon le sexe (homme sur le côté droit, femme sur le côté gauche), une orientation est/ouest des corps avec la tête à l'est, une inhumation des hommes à l'est de l'espace sépulcral et une inhumation des femmes à l'ouest de cet espace, dépôts d'objets et de crâne de zébu à proximité des défunts. La comparaison met simplement en lumière un changement de lieu et de milieu d'inhumation. L'abri funéraire utilisé antérieurement est remplacé par le cimetière villageois, le dépôt aérien du corps est remplacé par un dépôt souterrain. Un dépôt souterrain malgré tout particulier, puisque d'une certaine façon il recrée l'espace aérien : placé dans un espace vide, le défunt est isolé du contact direct de la terre par un dispositif protecteur.

La permanence des règles de dépôt des corps ainsi que la recréation de l'espace aérien suggèrent qu'il n'y a pas eu de modification profonde des gestes funéraires et ni probablement de leur signification. L'impression de continuité qui s'en dégage serait plutôt le fruit d'une transposition raisonnée des gestes funéraires d'un espace sépulcral vers un autre, répondant à de nouvelles contraintes matérielles (utilisation d'un espace de plein air). Il semblerait alors que les changements successifs n'aient pas induit d'évolution intrinsèque des rituels funéraires des communautés sakalava de la presqu'île de Narinda, l'intégration d'une nouvelle composante n'étant que l'acceptation de la perte d'une ancienne composante. Cette stabilité pourrait traduire une affirmation identitaire face au nouvel ordre sociopolitique, la cardinalisation de l'espace et de la division sexuelle étant des principes essentiels de l'idéologie sakalava appliqués systématiquement aux morts, mais aussi aux vivants et signalant des oppositions hiérarchiques, sociales, et rituelles [27,28].

\section{Discussion}

Décrypter les pratiques funéraires, d'un point de vue archéologique ou plus largement d'un point de vue social, revient à s'interroger sur ce qu'il convient de faire lors du décès d'un membre du groupe. Dans la séquence qui débute, certains gestes vont être nécessaires, ou obligatoires, c'est ce que nous avons appelé le « devoir-faire ", d'autres vont faire l'objet d'une prise de position, ou d'un choix ; il s'agit du « pouvoir-faire ». La norme, conçue comme une obligation, découle directement du premier terme : ce qui doit être fait. Elle n'intervient que secondairement dans ce qui peut être fait, en fixant les limites des choix : le «pouvoir-faire» est 
encadré par les interdits, ce qui ne doit pas être fait. Faire la part des obligations et des choix est un défi pour le préhistorien face aux données funéraires.

La norme, conçue comme un système, n'a un sens que dans une région donnée à une époque spécifique : autre temps, autre lieu, autre norme. Définir les limites de l'ensemble est la première difficulté. Dans le cas des allées sépulcrales de la culture Seine-Oise, nous sommes partis de la définition initiale de la culture archéologique, et nous avons testé la cohérence de l'ensemble ; coïncidence heureuse, l'entité qui se dégage finalement a les mêmes contours que la culture archéologique dans sa définition actuelle. Pour l'horizon Cerny du Bassin parisien, l'échantillon a été sélectionné sur un élément fort des pratiques funéraires. Nous avons ensuite examiné les variations de l'ensemble lorsqu'on se penche sur les autres caractères des pratiques funéraires. Il en résulte, dans ce cas, l'impossibilité de restituer une norme uniquement fondée sur des critères matériels. La situation, des deux côtés du fleuve Lerma, offre un bon contrepoint : un contexte culturel relativement homogène, deux normes funéraires clairement distinctes. L'opposition est telle qu'on ne peut la réduire à des expressions différentes ; il s'agit bien de deux systèmes différents dont on ne perçoit aucune interaction.

Définir l'ensemble initial, c'est essentiellement rechercher les obligations dues à tous les défunts. Une fois cette étape franchie, on doit s'interroger sur les variations de la norme : caractères qui n'ont qu'un lien indirect avec l'obligation, obligations qui ne s'appliquent qu'à une partie des défunts ou expressions du pouvoir-faire ? Les pratiques funéraires de la culture à céramique linaire permettent d'envisager comment le degré de liberté offert par la norme, examinée à une échelle large (la culture dans son aire d'extension maximale), prend la forme d'une règle au niveau régional. Un groupe humain relié à une entité plus large fait usage du " pouvoir-faire », qui devient à l'échelle locale un « devoir-faire». Le cas offert par le Natoufien n'est pas si différent. Les variations les plus importantes apparaissent comme le résultat d'options collectives, un « pouvoirfaire » collectif encadré par les interdits communs. Les choix individuels ne portent visiblement que sur le détail des pratiques et leur expression est strictement codifiée. Les sépultures du Néolithique moyen II en Bassin parisien montrent la difficulté à évoquer la norme lorsqu'on aborde qu'une frange infime de la population. La représentativité du corpus est au cœur de toute analyse. Peut-on seulement évoquer l'obligation dans un cas aussi extrême ? La tombe n'est-elle pas ellemême l'expression, pour le moins, d'un choix, du « pouvoirfaire $»$ ?

Si le préhistorien apprécie le découpage de l'espace et du temps en entités distinctes, cela ne l'exempte pas d'une réflexion sur l'évolution d'une entité, ou le passage de l'une à l'autre. La transformation d'une norme s'interprète-t-elle en termes de transgression, en termes d'une évolution des obligations, ou encore d'une refonte complète du système funéraire ? La nécropole d'Ifs montre le passage d'un système funéraire basé sur du « devoir-faire » à un système qui ouvre sur des « pouvoir-faire». L'exemple montre encore, à travers la continuité d'une norme sociale (le rôle prépondérant de certains sujets féminins), l'introduction de nouveaux choix funéraires. On peut envisager que la refonte complète de la norme funéraire est facilitée par la continuité de certaines règles sociales. Inversement, dans la presqu'île de Narinda, l'interaction de la société Sakalava avec la culture occidentale n'entraîne pas de changement majeur dans la norme funéraire. Dans cet exemple, l'évolution de la norme funéraire ne doit pas être forcément considérée comme un indicateur fidèle des transformations globales de la culture.

\section{Conclusion}

« Chacun de nous croit savoir d'une manière suffisante ce que c'est que la mort [...]» [1]. À l'issue de cet article, on pourrait faire la même constatation à propos de la notion de norme dans les pratiques funéraires. Ce travail collectif, suscité par des interrogations convergentes posées à des matériels chronologiquement et géographiquement divers, nous a emmenés bien plus loin que nous ne l'imaginions, bien au-delà des réponses que nous sommes à même de formuler. En premier lieu, il nous a fallu préciser le contenu des termes, et débrouiller un vocabulaire souvent vague, et toujours ambigu. C'est ainsi que l'emploi du terme « norme » nous est apparu pratique, car il nous délivre de la contrainte d'avoir à expliciter hypothétiquement (et en tout cas prématurément) des aspects dont nous ignorons tout, et qui d'ailleurs n'étaient pas nécessairement clairs pour les utilisateurs.

La norme interroge la culture, et la définition, forcément inadéquate, qu'en livre la préhistoire envisagée comme discipline. Qu'à une entité définie sur la base d'un assemblage matériel correspondent des choix funéraires cohérents demande une démonstration au cas par cas. Chercher des ensembles, c'est évidemment chercher des populations qui partagent un système de valeurs commun. Si nous prétendons que l'attitude face à la mort est pertinente de ce point de vue, il va sans dire qu'une position équivalente est soutenue par les spécialistes de la technologie, du style ou de l'habitat, etc.

La norme a un impact variable dans les témoignages archéologiques. Si les obligations n'ont que de faibles conséquences matérielles, elles nous échappent largement. Cependant, il va de soi que la recherche du devoir-faire ou du pouvoir-faire, dans les pratiques funéraires des sociétés préhistoriques, n'a de sens qu'en regard de l'interprétation qui en est faite, car ce sont bien les systèmes funéraires que nous poursuivons, in fine, et les idées qui les sous-tendent. 
Remerciements Nous remercions Michelle Elliott, Peter Macintyre et l'INRAP pour la traduction des résumés.

\section{Références}

1. Hertz R (1907) Contribution à une étude des représentations collectives de la mort. Ann Sociol 10: 48-137

2. Thomas LV (1985) Rites de mort. Fayard, Paris, 294 p

3. Testart A (2004) La servitude volontaire. 1. Les morts d'accompagnement. Errance, Paris, $263 \mathrm{p}$

4. Murphy EM (2008) Deviant burial in the archaeological record. Studies in funerary archaeology. Oxbow Books, Oxford, $244 \mathrm{p}$

5. Thomas LV (1984) Les rituels funéraires, Bulletin de la Société Française de Thanatologie, pp 60-61, repr. dans ID., Les Chairs de la mort. Corps, mort, Afrique, Paris, 2000, 137 p

6. Cannon A (1989) The historical dimension in mortuary expressions of status and sentiment. Curr Anthropol 30(4):437-58

7. Bosh-Guimpera P, Serra-Rafols J (1925-1927) Études sur le Néolithique et l'Ennéolithique de la France. Rev Anthropol 1925: 341-64; 1926:318-45; 1927:208-13

8. Chambon P, Salanova L (1996) Chronologie des sépultures du troisième millénaire dans le bassin de la Seine. Bull Soc Prehist Fr 93(1):103-18

9. Augereau A, Brunet P, Costa L, et al (2007) Le Néolithique récent dans le Centre-Nord de la France (3400/3300-2800/2700 av. J.-C.) : l'avenir du Seine-Oise-Marne en question. In: Evin J (ed) $\mathrm{XXVI}^{\mathrm{e}}$ Congrès préhistorique de France, Avignon, 21-25 sept 2004. SPF, Paris, pp 165-84

10. Chambon P, Mordant M, Pariat JG (2009) Sépultures du Néolithique moyen en Bassin parisien : le cas des architectures sépulcrales. In: Actes du XXVI ${ }^{\mathrm{e}}$ Colloque interrégional sur le Néolithique, Luxembourg 2003, Archaeologia Mosellana 7 (2007): pp 445-467

11. Duhamel P, avec la coll. de Fonton M, Carre H (1997) La nécropole monumentale de Passy (Yonne) : description d'ensemble et problèmes d'interprétation. In: Constantin C, Mordant D, Simonin D (eds) La Culture de Cerny, nouvelle économie, nouvelle société au Néolithique, actes du Colloque international de Nemours, mai 1994. APRAIF (Mémoires du musée de Préhistoire d'Ile-de-France 6), Nemours, pp 397-448

12. Chambon P (1997) La nécropole de Balloy les Réaudins, approche archéo-anthropologique. In: Constantin C, Mordant D, Simonin D (eds) La Culture de Cerny, Nouvelle économie, nouvelle société au Néolithique, actes du Colloque international de Nemours, mai 1994. APRAIF (Mémoires du musée de Préhistoire d'Ile-de-France 6), Nemours, pp 489-98

13. Pereira G (1999) Potrero de Guadalupe : anthropologie funéraire d'une communauté prétarasque du nord du Michoacán, Mexique, BAR. International series 816, Oxford, $249 \mathrm{p}$

14. Pereira G, Michelet D, Migeon G (2007) Cerro Barajas, Guanajuato. Archaeol Mex 87:77-82
15. Pereira G (2007), Le destin post-mortem du guerrier. Pratiques funéraires et statut social dans le Michoacán ancien, Mexique. In : Baray L, Brun P, Testart A (eds) Pratiques Funéraires et Société. Nouvelles approches en archéologie et en anthropologie sociale. Éditions Universitaires de Dijon, Dijon, pp 371-90

16. Sellier P (1987) Les sépultures de Mehrgarh : de l'analyse ostéologique à la reconstitution du rituel funéraire. Ann Fyssen 3:17-35

17. Thevenet C (2009) Les sépultures rubanées du Bassin parisien : recrutement sépulcral et implantation funéraire. In: Zeeb-Lanz A. (Hrsg) Krisen - Kulturalwandel - Kontinuitäten : Zum Ende der Bandkeramik in Mitteleuropa, Beiträge der Internationalen Tagung in Herxheim bei Landau (Pfalz) vom 14-17.06.2007. Internationale Archäologie, Arbeitsgemeinschaft, Symposium, Tagung, Kongress 10, Rahden/Westf, pp 111-27

18. Lichardus J, Lichardus-Itten M, Bailloud G, Cauvin J (1985) La Protohistoire de l'Europe. Le Néolithique et le Chalcolithique, Nouvelle Clio $\mathrm{n}^{\mathrm{o}} 1$ bis, Presses Universitaires de France, Paris, 640p

19. Valla FR (1999) The Natufian: A coherent thought? In: Davies W, Charles R (eds) Dorothy Garrod and the progress of the Palaeolithic: studies in the Prehistoric archaeology of the near east and Europe. Oxbow, Oxford, pp 224-41

20. Bocquentin F (2003) Pratiques funéraires, paramètres biologiques et identités culturelles au Natoufien : une analyse archéoanthropologique. Thèse de Doctorat en Anthropologie Biologique (unpublished). Université Bordeaux 1, Talence, 629 p. http://grenet. drimm.u-bordeaux1.fr/pdf/2003/BOCQUENTIN_FANNY_2003.pdf

21. Pariat JG (2007) Des morts sans tombe ? BAR International Series 1683, Oxford, $195 \mathrm{p}$

22. Le Goff E, Auxiette G, Le Goff I, et al (2006) Manifestation et pratiques cultuelles au son des habitats et du territoire agraire laténien de la "ZAC Object'Ifs Sud » à Ifs (Calvados) : un exemple de l'Ouest de la Gaule. In: Barral P, Daubigney A, Dunning C, et al (eds) L'âge du Fer dans l'arc jurassien et ses marges. Dépôts, lieux sacrés et territorialités à l'âge du Fer. Actes du XXIX $^{\mathrm{e}}$ Colloque international de l'AFEAF; Bienne, 5-8 mai 2005. Presses Universitaires de Franche-Comté (annales littéraires; série "Environnement, sociétés et archéologie »), Besançon, pp 565-70

23. Leroi-Gourhan A (1965) Le geste et la parole, t. II : la mémoire et les rythmes. Albin Michel, Paris, 285 p

24. Kaufmann JC (2007) Ego. Pour une sociologie de l'individu, coll. Pluriel, Hachette, Paris, $275 \mathrm{p}$

25. Decary R (1962) La mort et les coutumes funéraires à Madagascar, Maisonneuve Larose, Paris, 306 p

26. Valentin F, Ramanivosoa B, Gommery D, et al (2006) Les abris sépulcraux de la presqu'île de Narinda (Province de Mahajanga, Madagascar). Afrique. Arch Arts 4:7-22

27. Ballarin MP (2000) Les reliques royales à Madagascar, source de légitimation et enjeux de pouvoir $\left(\mathrm{XVIII}^{\mathrm{e}}-\mathrm{XX}^{\mathrm{e}}\right)$, Karthala, Paris, $470 \mathrm{p}$

28. Godefroit S (1998) À l'ouest de Madagascar : les Sakalava du Menabe, Karthala, Orstom, Paris, 529 p 